\title{
Convolutional neural network with hyperparameter tuning for brain tumor classification
}

\author{
Agus Eko Minarno*1, Mochammad Hazmi Cokro Mandiri², Yuda Munarko ${ }^{3}$, Hariyady ${ }^{4}$ \\ Universitas Muhammadiyah Malang, Indonesia ${ }^{1,2}$
}

\section{Article Info}

Keywords:

Brain Tumor, MRI, Convolutional Neural

Network, Hyperparameter Tuning

\section{Article history:}

Received: February 16, 2021

Accepted: March 18, 2021

Published: May 31, 2021

\section{Cite:}

Minarno, A. E., Mochammad Hazmi Cokro Mandiri, Munarko, Y. ., \& Hariyady, H. (2021). Convolutional Neural Network with

Hyperparameter Tuning for Brain Tumor Classification. Kinetik: Game Technology, Information System, Computer Network,

Computing, Electronics, and Control, 6(2).

https://doi.org/10.22219/kinetik.v6i2.1219

\begin{abstract}
Brain tumor has been acknowledged as the most dangerous disease through all its circles. Early identification of tumor disease is considered pivotal to identify the spread of brain tumors in administering the appropriate treatment. This study proposes a Convolutional Neural Network (CNN) method to detect brain tumor on MRI images. The 3264 datasets were undertaken in this study with detailed images of Glioma tumor (926 images), Meningioma tumors (937 images), pituitary tumors (901 images), and other with no-tumors (500 images). The application of CNN method combined with Hyperparameter Tuning is proposed to achieve optimal results in classifying the brain tumor types. Hyperparameter Tuning acts as a navigator to achieve the best parameters in the proposed CNN model. In this study, the model testing was conducted with three different scenarios. The result of brain tumor classification depicts an accuracy of $96 \%$ in the third (3rd) model testing scenario.
\end{abstract}

*Corresponding author

Agus Eko Minarno

E-mail address:

aguseko@umm.ac.id

\section{Introduction}

Brain tumor disease has been regarded as one of the most dangerous and deadly cancers among adults and children. Early identification and classification of brain tumors into specific classes is deemed essential to administer the effective treatment [1]. Diagnosis of a brain tumor is typically based on image data analysis of brain tumor. Accurate analysis of brain tumor images serves as the primary step in determining the condition of a patient with a brain tumor [2].

In general, brain tumor is divided into two types of benign and malignant tumor. Benign tumor is identified when it does not spread to other parts of the brain; contrastingly, malignant tumor is signified when it spreads to other parts of the brain or outside of the brain [3]. The classification of brain tumor is recognized by the grades or levels which includes: grade I and grade II classified as low level brain tumor; grade III and grade IV classified as high level brain tumor [4][5]. This study engages the three types of brain tumors, including: glioma tumors, meningioma tumors, and pituitary tumors. Glioma tumor is recognized as a tumor that attacks the brain and spinal cord; meningioma tumor is distinguished as a tumor that grows on the lining of the brain and spinal cord; and pituitary tumor is identified as a tumor that appears on the pituitary gland [6].

Hence, early diagnosis of a brain tumor is required in detecting the condition of a patient. The diagnosis is performed to determine the spread of brain tumor and to administer the appropriate treatment [7]. Magnetic Resonance Imaging (MRI) technology is broadly accredited to provide images of the organ structure by utilizing magnetic fields, which are documented via a computer [8]. The results of the MRI process are in the form of images, employed to classify brain tumor status [9]. The experience of radiologists in analyzing the results of MRI images, however, leads to a relatively extended delay to perform the procedure [10].

Deep learning (DL) is a branch of machine learning based on a system development to study multiple level representation by creating hierarchical features, where higher level is determined from lower level and similar lower level features could assist in determining numerous higher level features [11]. Convolutional Neural Network is an algorithm in deep learning that focuses on the problem of classifying an image data [12]. Hence, CNN method has the ability to study, represent, and classify objects. Further, CNN method has a sequence initiated from the convolutional layer, pooling layer and fully connected layer which functions to change 2D features from the previous layer to 1D vectors for the classification process [13].

Previous research in 2019 conducted by A. Yang et al. [14] proposed a classification system for benign tumor and malignant tumor using the Convolutional Neural Network method by applying the pretrained Xception and Dense Net models to improve the accuracy of CNN algorithm. Similarly in 2020, X. Liu et al. [15] performed the classification

Cite: Minarno, A. E., Mochammad Hazmi Cokro Mandiri, Munarko, Y. ., \& Hariyady, H. (2021). Convolutional Neural Network with Hyperparameter Tuning for Brain Tumor Classification. Kinetik: Game Technology, Information System, Computer Network, Computing, Electronics, and Control, 6(2). https://doi.org/10.22219/kinetik.v6i2.1219 
of glioblastoma tumor by utilizing CNN method to prove that CNN method was deemed reliable and accurate in the tumor classification process. In 2020, P. Nagaraj et al. [16] performed a classification of Meningioma, Glioma, and Pituitary brain tumors with 3064 images. In this study, the 2 experiments were undertaken with the first experiment to classify the brain tumor status and the second experiment to classify the tumor grade. In 2018, N. Abiwinanda et al. [17] conducted relevant research related to brain tumor which had 3 classes with 3064 images. In this study, CNN algorithm was performed for classification indicating an accuracy of $94.68 \%$. Additionally in 2020 , L.Bhaiya et al. [18] reported the classification of Meningioma, Glioma, and Pituitary MRI images employing CNN method. The process of CNN method has implemented the pretrained model from VGG16 and VGG19, which is balanced by transfer learning. The accuracy obtained with the VGG16 model is $84.59 \%$, while the accuracy obtained by the VGG19 model is $86.70 \%$.

Based on the problem description as mentioned earlier in [17][18], this study proposes a classification of brain tumor status by employing Convolutional Neural Network method combined with hyperparameter tuning. More specifically, this study aims to classify brain tumor status images with a total dataset of 3264 files in "jpg" extension. This study adds the novel notion compared with the previous studies in terms of larger number of datasets, having four classes of brain tumor status which include: glioma, meningioma, pituitary, and no tumor (normal) and develops a new architectural model based on Convolutional Neural Network. In the preprocessing stage, data normalization and augmentation were undertaken on the dataset. At the implementation stage of the proposed CNN model, the addition of the hyperparameter tuning process was performed to optimize the established model as a contribution to this study.

\section{Research Method}

This section discusses the employed research methods for brain tumor classification (types of brain tumor) by utilizing one of the branches of deep learning, which is Convolutional Neural Network. In this paper, a combination of the CNN method with Hyperparameter Tuning is presented to obtain optimal parameters for the proposed model.

\subsection{Dataset}

In this study, the employed dataset includes brain tumor data, recruited from a website acknowledged as Kaggle consisting of 3264 files [19]. The dataset has 4 types of classes, which comprise: Glioma tumors of 926 images, Meningioma tumors of 937 images, Pituitary tumors totaling of images, and other tumor numbers of 500 images. The following is an example image of the axial, sagittal and coronal planes in each brain tumor data class as illustrated in Figure 1.

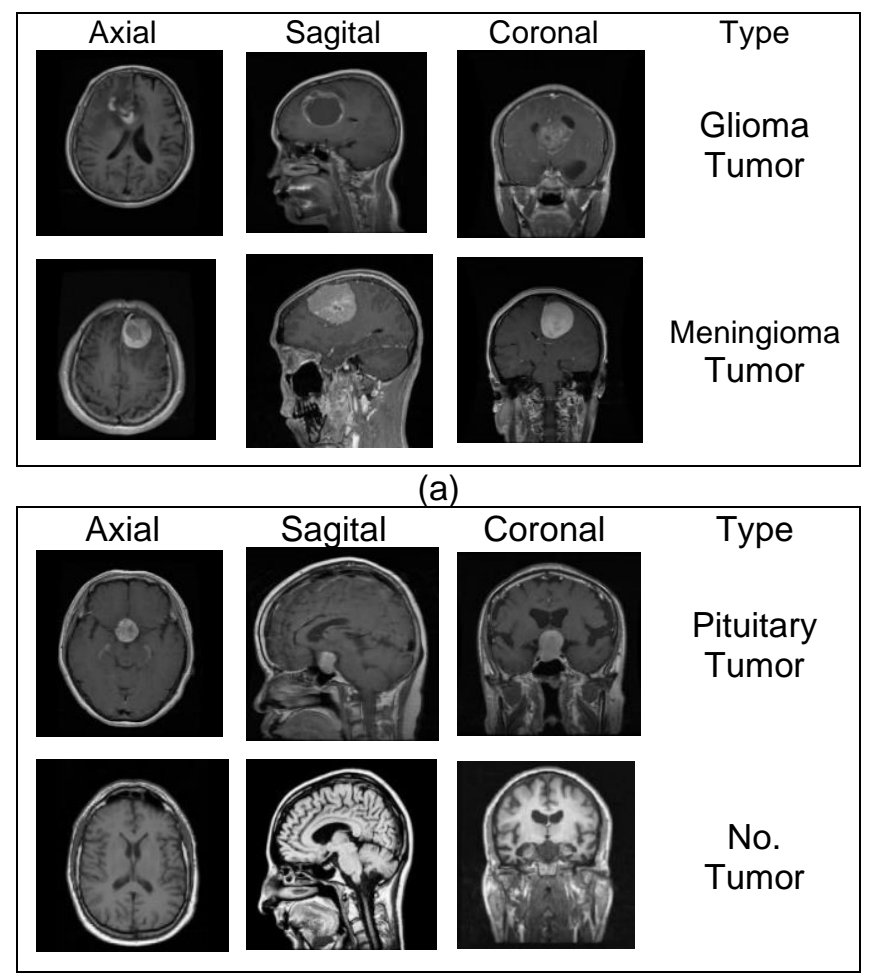

(b)

Figure 1. Sample of MRI Images of Brain Tumor in Each Class

(c) 2021 The Authors. Published by Universitas Muhammadiyah Malang

This is an open access article under the CC BY SA license. (https://creativecommons.org/licenses/by-sa/4.0/) 


\subsection{Preprocessing Data}

In this study, data preprocessing was conducted to obtain images of brain tumor diseases, starting from the stage of reallocating the dataset into $80 \%$ train data, $10 \%$ test data and $10 \%$ validation data. The next step is progressed to change the image size from $512 \times 512$ pixels to $128 \times 128$ pixels [20], to equalize the dimensions of each image in each class of brain tumors. Then normalization is further conducted by utilizing the encoding label on selected images which is valuable in modifying the image form value to binary for each brain tumor class.

\subsection{Augmentation}

At this stage, data augmentation is performed to amplify the amount of data by altering the original image to be recognized as a different image. Augmentation could improve the performance of classification results [21]. Additionally, this process could produce results for the exceeding experiments which require additionally extra data [22]. The augmentation techniques used in this process include: rotation, zoom, shift, and flip.

\subsection{Implementation of the CNN Model}

In this study, the application of CNN model architecture is performed on the brain tumor dataset to classify tumor types, including No Tumor, Glioma, Meningioma and Pituitary. In the process of creating the proposed CNN model, the addition of hyperparameter tuning stages is conducted to locate a model with the best parameter; thus achieving a superior performance [23]. The proposed model in this study includes a convolutional layer, a pooling layer, a dropout layer, and a dense layer. The number of dropout layers will be added after the pooling layer and before the next convolutional layer, performed to prevent overfitting and to improve the model performance [22]. The following chart illustrates the process of CNN model architecture application on the brain tumor dataset initiated from the preprocessing stage, the model design stage, the hyperparameter tuning process stage and the model evaluation until achieving the model accuracy as summarized in Figure 2.

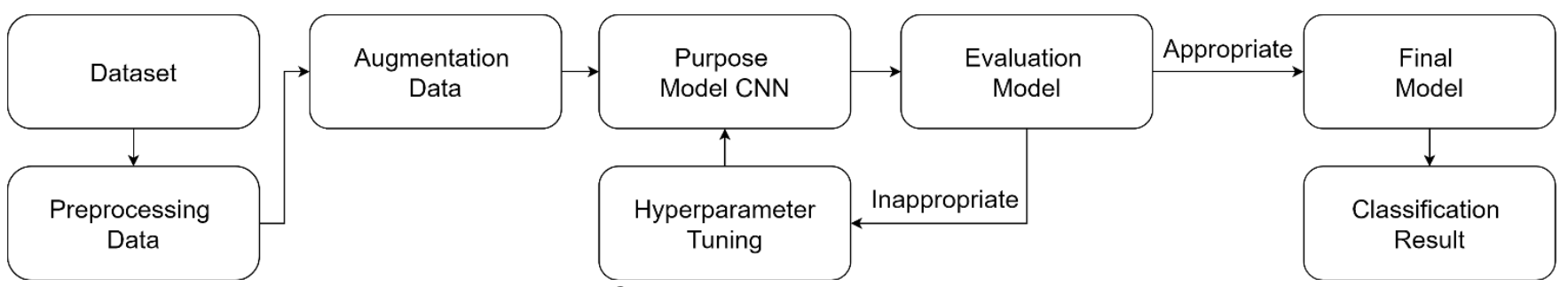

Figure 2. CNN Model Implementation Design

In this study, the design of CNN model architecture has been constructed to acquire the accuracy of brain tumor classification process. The design results of CNN model architecture is illustrated in Table 1.

Table 1. CNN Model Design

\begin{tabular}{cccc}
\hline Layer & Filter & Kernel_size & Activation \\
\hline $\begin{array}{c}\text { Input (128,128) } \\
\text { Conv2D }\end{array}$ & 64 & 5.5 & Relu \\
$\begin{array}{c}\text { MaxPooling2D } \\
\text { Dropout } \\
\text { Conv2D }\end{array}$ & 128 & 3,3 & Relu \\
$\begin{array}{c}\text { MaxPooling2D } \\
\text { Dropout } \\
\text { Conv2D }\end{array}$ & 128 & 3,3 & Relu \\
$\begin{array}{c}\text { MaxPooling2D } \\
\text { Dropout } \\
\text { Conv2D }\end{array}$ & 128 & 3,3 & Relu \\
$\begin{array}{c}\text { MaxPooling2D } \\
\text { Dropout } \\
\text { Conv2D }\end{array}$ & 256 & 3,3 & Relu \\
MaxPooling2D \\
Dropout
\end{tabular}

Cite: Minarno, A. E., Mochammad Hazmi Cokro Mandiri, Munarko, Y. ., \& Hariyady, H. (2021). Convolutional Neural Network with Hyperparameter Tuning for Brain Tumor Classification. Kinetik: Game Technology, Information System, Computer Network, Computing, Electronics, and Control, 6(2). https://doi.org/10.22219/kinetik.v6i2.1219 


\begin{tabular}{|c|c|c|c|}
\hline \multicolumn{4}{|l|}{ Flatten } \\
\hline Dense & 1024 & - & Relu \\
\hline \multicolumn{4}{|l|}{ Dropout } \\
\hline Dense & 4 & & Softmax \\
\hline
\end{tabular}

\subsection{Hyperparameter Tuning}

Hyperparamater Tuning provides an optimal parameter search method for the proposed Convolutional Neural Network model. CNN combined with Hyperparameter Tuning requires to set the parameters, such as: kernel size, steps, number of channels, and number of dropouts [24]. Hyperparameter Tuning provides the best combination of parameters to the model demonstrating maximum results [25]. In this study, Hyperparameter Tuning is selected to provide the best parameter through the proposed comparison parameters. The comparative parameters are illustrated in Table 2 as follows.

Table 2. CNN Comparison Parameters

\begin{tabular}{cc}
\hline Parameter & Comparative Value \\
\hline Dropout & $0.2,0.5$ \\
Dense Layer & 1024,512 \\
Optimizer & Adam, Adamax, RMSprop, SGD \\
\hline
\end{tabular}

\section{Results and Discussion}

In this study, the three scenario models were examined with different parameters to determine the effect of Hyperparameter Tuning on the developed CNN model. The following Table 3 provides the Model Testing Scenario and descriptions of each scenario.

Table 3. Description of Model Testing Scenario

\begin{tabular}{cc}
\hline Scenario & Description \\
\hline Model 1 & $\begin{array}{c}\text { The proposed CNN model is in Table 1. Design } \\
\text { of the CNN Model }\end{array}$ \\
Model 2 & $\begin{array}{c}\text { Model with the 2nd Best Tuning } \\
\text { Hyperparameter Results Parameters } \\
\text { Model with the 1st best parameter tuning } \\
\text { hyperparameter results }\end{array}$ \\
\hline
\end{tabular}

\subsection{Model Testing Scenario 1}

Model Testing Scenario 1 contains tests on the model design that has been formed in Table 1. In this scenario, training data is conducted with Adam's optimizer with a learning rate of 0.0014 , an epoch of 100 , categorical classification and Relu activation. Following are the results of the accuracy and loss plot in the scenario model 1 as illustrated in Figure 3.
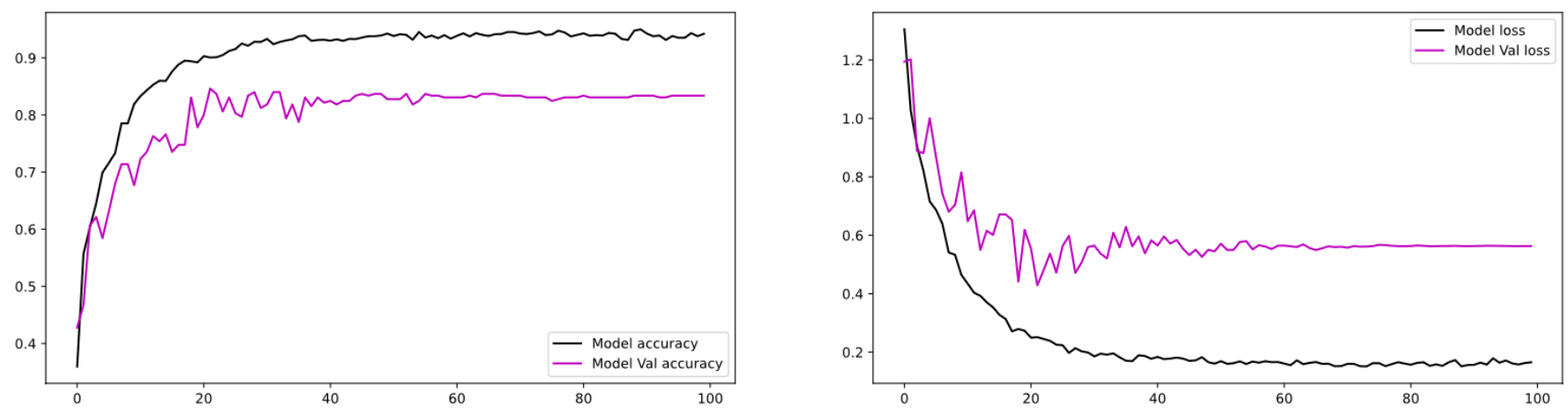

Figure 3. Scenario Model 1 Results

\subsection{Model Testing Scenario 2}

The model scenario 2 contains testing on the model design with the second-best parameter resulting from the Hyperparameter Tuning that has been performed in the model design. The results of the best parameters from Hyperparameter Tuning process are summarized in Table 4 as follows. 
Kinetik: Game Technology, Information System, Computer Network, Computing, Electronics, and Control

\begin{tabular}{cccc}
\multicolumn{4}{c}{ Table 4. Hyperparameter Tuning Results } \\
\hline Parameter & Optimizer & Dropout & Dense Layer \\
\hline 1 & RMSprop & 0.2 & 512 \\
2 & Adam & 0.2 & 512 \\
3 & Adam & 0.2 & 1025 \\
\hline
\end{tabular}

In this scenario, training data is performed with the second-best parameter of Hyperparameter Tuning, which includes: Adam's optimizer, a dropout of 0.2, and a dense layer of 512.The following plot results for accuracy and loss in model 2 are illustrated in Figure 4 as follows.
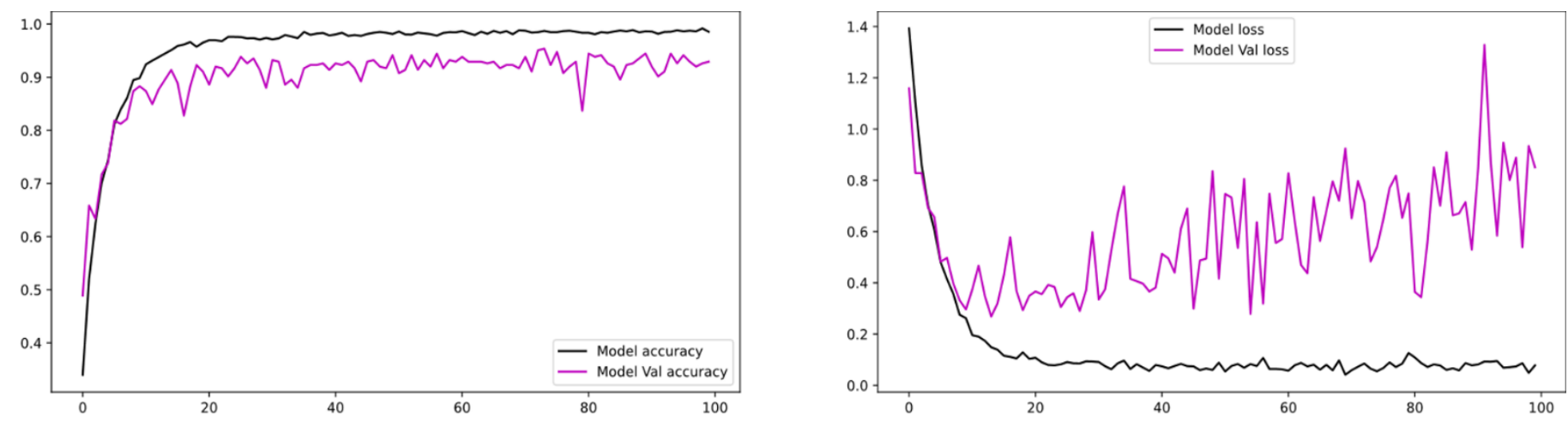

Figure 4. Scenario Model 2 Results

\subsection{Model Testing Scenario 3}

Model Testing Scenario 3 consists of testing on the model design with the best parameter 1 resulting from the performed Hyperparameter Tuning. In this scenario, data training is undertaken with the first best parameter which includes: RMSprop optimizer, a dropout of 0.2 , and a dense layer of 512. The following plot results for accuracy and loss in model scenario 3 are illustrated in Figure 5.
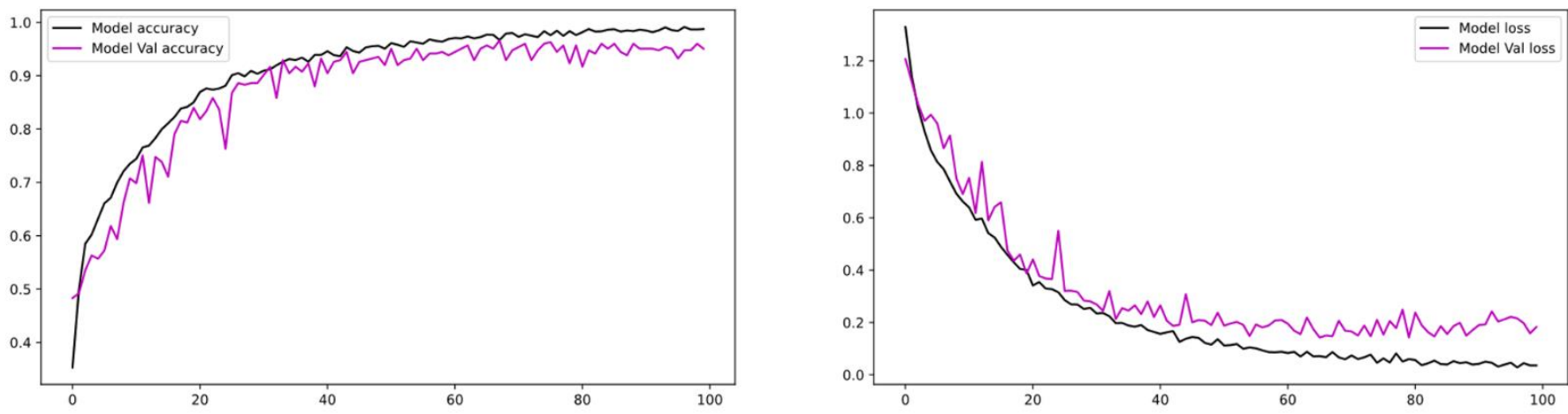

Figure 5. Scenario Model 3 Results

\subsection{Results and Model Evaluation}

The test results of each performed model scenario indicated that there was a significant effect of applying Hyperparameter Tuning in determining the best parameters for the proposed model in terms of accuracy. Results of the accuracy testing for the three model scenarios are summarized in Table 5 as follows.

Table 5. Model Testing Results

\begin{tabular}{ccccc}
\hline Scenario & Accuracy & Precission & Recall & F1-Score \\
\hline Model 1 & 86 & 87 & 86 & 86 \\
Model 2 & 91 & 91 & 91 & 91 \\
Model 3 & 96 & 96 & 96 & 96 \\
\hline
\end{tabular}

In this study, a model evaluation was performed against the previous studies. The details from comparisons to previous studies are illustrated in Table 6 as follows.

Cite: Minarno, A. E., Mochammad Hazmi Cokro Mandiri, Munarko, Y. ., \& Hariyady, H. (2021). Convolutional Neural Network with Hyperparameter Tuning for Brain Tumor Classification. Kinetik: Game Technology, Information System, Computer Network, Computing, Electronics, and Control, 6(2). https://doi.org/10.22219/kinetik.v6i2.1219 


\section{Table 6. Model Testing Results}

\begin{tabular}{cccc}
\hline Author & Dataset & Model & Accuracy \\
\hline L.Bhaiya et al. & Grade 3 Brain Tumor & VGG16, VGG19 & $84.59 \%, 86.70 \%$ \\
Model 1 & Grade 4 Brain Tumor & CNN & $86 \%$ \\
Model 2 & Grade 4 Brain Tumor & CNN & $91 \%$ \\
Model 3 & Grade 4 Brain Tumor & CNN & $96 \%$ \\
\hline
\end{tabular}

\section{Conclusion}

Early detection of brain tumors has been considered pivotal in administering appropriate medical treatment. This study aims to classify brain tumors into four classes, which includes: no tumor, glioma, meningioma, and pituitary. In this study, the three scenario models were performed and the best results were obtained in the $\left(3^{\text {rd }}\right)$ third model with an accuracy of $96 \%$, while the best parameters were obtained from the results of Hyperparameter Tuning in the proposed model. Future research is encouraged to discover novel methods for brain tumor classification and to navigate new parameters for potential model.

\section{References}

[1] M. Sajjad, S. Khan, K. Muhammad, W. Wu, A. Ullah, and SW Good, "Multi-grade brain tumor classification using deep CNN with extensive data augmentation," J. Comput. Sci., vol. 30, pp. 174-182, 2019. https://doi.org/10.1016/j.jocs.2018.12.003

[2] MA Khan et al., "Brain tumor detection and classification: A framework of marker-based watershed algorithm and multilevel priority features selection," Microsc. Res. Tech., Vol. 82, no. 6, pp. 909-922, 2019. https://doi.org/10.1002/jemt.23238

[3] A. Minz and C. Mahobiya, "MR image classification using adaboost for brain tumor type," Proc. - 7th IEEE Int. Adv. Comput. Conf. IACC 2017, pp. 701-705, 2017. https://doi.org/10.1109/IACC.2017.0146

[4] G. Mohan and MM Subashini, "MRI based medical image analysis: Survey on brain tumor grade classification," Biomed. Signal Process. Control, vol. 39, pp. 139-161, 2018. https://doi.org/10.1016/j.bspc.2017.07.007

[5] M. Toğaçar, B. Ergen, and Z. Cömert, "Tumor type detection in brain MR images of the deep model developed using hypercolumn technique, attention modules, and residual blocks," Med. Biol. Eng. Comput., 2020. https://doi.org/10.1007/s11517-020-02290-x

[6] S. Deepak and PM Ameer, "Brain tumor classification using deep CNN features via transfer learning," Comput. Biol. Med., vol. 111, no. June, pp. 103345, 2019. https://doi.org/10.1016/j.compbiomed.2019.103345

[7] A. Pashaei, H. Sajedi, and N. Jazayeri, "Brain tumor classification via convolutional neural network and extreme learning machines," 2018 8th Int. Conf. Comput. Knowl. Eng. ICCKE 2018, no. Iccke, pp. 314-319, 2018. https://doi.org/10.1109/ICCKE.2018.8566571

[8] H. Shahamat and M. Saniee Abadeh, "Brain MRI analysis using a deep learning based evolutionary approach," Neural Networks, vol. 126, pp. 218-234, 2020. https://doi.org/10.1016/j.neunet.2020.03.017

[9] N. Noreen, S. Palaniappan, A. Qayyum, I. Ahmad, M. Imran, and M. Shoaib, "A Deep Learning Model Based on Concatenation Approach for the Diagnosis of Brain Tumor," IEEE Access, vol. 8, pp. 55135-55144, 2020. https://doi.org/10.1109/ACCESS.2020.2978629

[10] Z. Fan, F. Xu, X. Qi, C. Li, and L. Yao, "Classification of Alzheimer's disease based on brain MRI and machine learning," Neural Comput. Appl., vol. 32, no. 7, pp. 1927-1936, 2020. https://doi.org/10.1007/s00521-019-04495-0

[11] H. Mohsen, E.-SA El-Dahshan, E.-SM El-Horbaty, and A.-BM Salem, "Classification using deep learning neural networks for brain tumors," Futur. Comput. J. Informatics, vol. 3, no. 1, pp. 68-71, 2018. https://doi.org/10.1016/j.fcij.2017.12.001

[12] S. Kumar and DP Mankame, "Optimization driven Deep Convolution Neural Network for brain tumor classification," Biocybern. Biomed. Eng., vol. 40, no. 3, pp. 1190-1204, 2020. https://doi.org/10.1016/j.bbe.2020.05.009

[13] D. Ravi et al., "Deep Learning for Health Informatics," IEEE J. Biomed. Heal. Informatics, vol. 21, no. 1, pp. 4-21, 2017. https://doi.org/10.1109/JBHI.2016.2636665

[14] A. Yang, X. Yang, W. Wu, H. Liu, and Y. Zhuansun, "Research on feature extraction of tumor image based on convolutional neural network," IEEE Access, vol. 7, pp. 24204-24213, 2019. https://doi.org/10.1109/ACCESS.2019.2897131

[15] X. Liu, X. Zhou, and X. Qian, "Transparency-guided ensemble convolutional neural network for the stratification between pseudoprogression and true progression of glioblastoma multiform in MRI," J. Vis. Commun. Image Represent., vol. 72, no. August, pp. 102880, 2020. https://doi.org/10.1016/j.jvcir.2020.102880

[16] P. Nagaraj, V. Muneeswaran, L. Veera Reddy, P. Upendra, and M. Vishnu Vardhan Reddy, "Programmed Multi-Classification of Brain Tumor Images Using Deep Neural Network," Proc. Int. Conf. Intell. Comput. Control Syst. ICICCS 2020, pp. 865-870, 2020. https://doi.org/10.1109/ICICCS48265.2020.9121016

[17] N. Abiwinanda, M. Hanif, ST Hesaputra, A. Handayani, and TR Mengko, Brain Tumor Classification Using Convolutional Neural Network, vol. 68, no. 1. Springer Singapore, 2018.

[18] LP Bhaiya and VK Verma, "Classification of MRI Brain Images Using Neural Networks," vol. 2, no. 5, pp. 751-756, 2012.

[19] S. Bhuvaji, A. Kadam, P. Bhumkar, and S. Dedge, "Brain Tumor Classification (MRI) | Kaggle, "2020.

[20] M. Agarwal, SK Gupta, and KK Biswas, "Development of Efficient CNN model for Tomato crop disease identification," Sustain. Comput. Informatics Syst., vol. 28, p. 100407, 2020. https://doi.org/10.1016/j.suscom.2020.100407

[21] X. Xiao, M. Yan, S. Basodi, C. Ji, and Y. Pan, "Efficient Hyperparameter Optimization in Deep Learning Using a Variable Length Genetic Algorithm," arXiv, 2020.

[22] YD Zhang, C. Pan, J. Sun, and C. Tang, "Multiple sclerosis identification by convolutional neural network with dropout and parametric ReLU," J. Comput. Sci., vol. 28, pp. 1-10, 2018. https://doi.org/10.1016/j.jocs.2018.07.003

[23] M. Frid-Adar, I. Diamant, E. Klang, M. Amitai, J. Goldberger, and H. Greenspan, "GAN-based synthetic medical image augmentation for increased CNN performance in liver lesion classification," Neurocomputing, vol. 321, pp. 321-331, 2018. https://doi.org/10.1016/j.neucom.2018.09.013

[24] MP Ranjit, G. Ganapathy, K. Sridhar, and V. Arumugham, "Efficient deep learning hyperparameter tuning using cloud infrastructure: Intelligent distributed hyperparameter tuning with Bayesian optimization in the cloud," IEEE Int. Conf. Cloud Comput. CLOUD, vol. 2019-July, pp. 520522, 2019. https://doi.org/10.1109/CLOUD.2019.00097

[25] WY Lee, SM Park, and KB Sim, "Optimal hyperparameter tuning of convolutional neural networks based on the parameter-setting-free harmony search algorithm," Optics (Stuttg)., vol. 172, no. July, pp. 359-367, 2018. https://doi.org/10.1016/j.ijleo.2018.07.044 